\title{
WHOLE GENOME ANALYSIS BY ARRAY-BASED COMPARATIVE GENOMIC HYBRIDIZATION IN PATIENTS WITH CONGENITAL MALFORMATIONS
}

\author{
Dimova $\mathrm{I}^{1}$, Vazharova $\mathrm{R}^{1}$, Nikolova $\mathrm{D}^{1}$, Tincheva $\mathrm{R}^{2}$, \\ Nesheva $\mathrm{D}^{1}$, Uzunova $\mathrm{Y}^{3}$, Toncheva $\mathrm{D}^{1, *}$
}

*Corresponding Author: Professor Draga Toncheva, Department of Medical Genetics, Medical University Sofia, 2, Zdrave str., 1431 Sofia, Bulgaria; Tel./Fax: +359-2-952-0357; E-mail: dragatoncheva@yahoo.com

\begin{abstract}
Congenital malformations present at delivery of an infant are due to genetic or non genetic factors and occur in $15-20 \%$ of stillborn children. Most can be diagnosed prenatally by ultrasound examination, but some can only be diagnosed after birth. Seven to $10 \%$ of infants with abnormal phenotype have numerical or structural chromosomal abnormalities that require identification for accurate diagnosis and genetic counseling. Molecular-cytogenetic and array-based techniques have enabled screening at higher resolution for congenital anomalies that result from genomic imbalances. We have examined four children with congenital anomalies, with or without mental retardation, of unclear etiology. In one child, we detected a deletion (about $28 \mathrm{Mb}$ ) of the region 18q21.1-18q23, in mosaic form. This abnormality was missed in a routine cytogenetic examination. We detected different polymorphic copy number variations (CNVs) in the other children. We conclude that array-based comparative genomic hybridization $(\mathrm{CGH})$ is a powerful diagnostic tool for the detection of low level mosaicism.
\end{abstract}

1 Department of Medical Genetics, Medical University Sofia, Sofia, Bulgaria

2 Department of Pediatrics, Section Clinical Genetics, Medical University Sofia

3 Department of Urology, Medical University Sofia, Sofia, Bulgaria
Key words: Congenital malformations; Genomic imbalances; DNA microarrays; Mosaicism

\section{INTRODUCTION}

In Bulgaria, about 2,100 children are born with congenital malformations annually. Most malformations are present at delivery and are due to genetic and non genetic factors [1]. They occur at a relatively constant population frequency of $2-4 \%$ independent of race, culture and social-economic environment, and are observed in $15-20 \%$ of stillborn infants. About $60 \%$ of infants with malformations are diagnosed during the first month of life and about $80 \%$ by the end of the third month. With the introduction of ultrasonography in the routine clinical examination of pregnancy, severe congenital anomalies can be diagnosed antenatally [2], but many congenital defects cannot be diagnosed during pregnancy. About $20-25 \%$ of congenital malformations are considered to be multifactorial, and $10-13 \%$ are due to environmental factors such as infection, drugs or maternal disease $[3,4]$. The rest have no known cause. Chromosome abnormalities have long been recognized as an important cause of multiple malformation syndromes, $7-10 \%$ of infants with abnormal phenotype having numerical or structural chromosomal aberrations [5]. Their identification is important for accurate diagnosis and genetic counseling. 
The most frequent structural chromosomal abnormalities include unbalanced translocation, duplication, deletion, isochromosomes, ring chromosomes and micro-structural aberrations [5]. Some are so minor that they cannot be diagnosed by classical karyotyping. These include microdeletions or microduplications, some of them being associated with recognizable syndromes. The affected regions on the chromosome include genes which may contribute separately or simultaneously to the characteristics of the phenotype [6]. We have studied four patients with normal karyotypes who suffered from congenital malformations with or without mental retardation, by means of array-based comparative genomic hybridization (CGH) to detect microstructural genomic abnormalities and/or polymorphic regions.

\section{CASE HISTORIES}

Patient 1 was a male aged 3 years and 4 months with a normal mental development. He had a developed asymmetry of the lower limbs, left-sided microdactyly and hyperthelorism. He had undergone two operations for resection of tumors in the right kidney and the liver. The routine cytogenetic analysis did not reveal any chromosomal abnormalities and showed a normal male karyotype $(46, \mathrm{XY})$.

Patient 2 was a female aged 9 years and 4 months with a severe mental retardation, speech deficiency and low intelligence (IQ 25). She had facial dysmorphism, congenital dysplasia of the pelvic joint, contractures of the ankle joints and epilepsy. There was no evidence of metabolic disorder. Cytogenetic analysis showed a normal female karyotype $(46, \mathrm{XX})$. The diagnosis was "mental retardation of unclear etiology."

Patient 3 was a male aged 2 years and 7 months with severe mental retardation, lack of speaking ability, hypotonic muscles, microcephaly and facial dysmorphism. There were no anomalies of the heart, kidneys and gastro-intestinal tract. The karyotype was normal $(46, \mathrm{XY})$. The diagnosis was "malformative syndrome of unclear etiology."

Patient 4 was a female aged 13 years and 8 months with a severe mental retardation (IQ 5), epilepsy, spastic paresis of limbs and hyperactivity. There was facial dysmorphism - hypertelorism and convergent strabismus, but no organ anomalies. The karyotype was normal (46,XX). The diagnosis was "malformative syndrome of unclear etiology."

\section{MATERIALS AND METHODS}

The study was approved by the Ethics Committee of the Medical University of Sofia, Sofia, Bulgaria. The parents of the children provided their informed consent. Peripheral blood was taken for genetic diagnostic testing.

Chromosome Analysis. G-banded chromosomes were prepared from whole blood samples using standard laboratory protocol.

DNA Extraction. DNA was extracted from blood samples by phenol-chloroform. The yield and purity for protein/RNA were estimated by a Nanodrop 1000 spectrophotometer (Thermo Scientific, Wilmington, DE, USA). The DNA was checked on a $1 \%$ agarose gel: DNA of high molecular weight $(>50 \mathrm{~kb})$ indicated it was suitable for use.

Genomic Arrays. We have used genomic array CytoChip (BlueGnome, Cambridge, Cambridgeshire, UK), covering the entire genome by bacterial artificial chromosome (BAC) clones at a median $565 \mathrm{~kb}$, a resolution optimized to detect pathogenic imbalances while minimizing polymorphisms. In addition, sub-telomeric clones were included at a median $250 \mathrm{~kb}$ resolution, thus allowing reliable detection of mosaicism. The BAC clones of 90 known genetic conditions at a median $100 \mathrm{~kb}$ resolution were also included in the chip. This resulted in an average density of 1 clone $/ 0.5$ $\mathrm{Mb}$.

Probe Labeling, Hybridization, Image Capture and Data Analysis. Test- and sex-matched reference genomic DNA (400 ng) was labeled by random-priming, using a fluorescent labeling system (BlueGnome). The labeled products were purified by AutoSeq ${ }^{\mathrm{TM}}$ G50 columns (Amersham Pharmacia Biotech Inc., Piscataway, NY, USA), and the incorporation of dyes was evaluated by the spectrophotometer (Nanodrop 1000, Thermo Scientific). Incorporation in the range of $6-15 \mathrm{pmol} / \mu \mathrm{L}$ and a DNA yield of $180-325 \mathrm{ng} / \mu \mathrm{L}$ were considered suitable for further analysis. A mix of Cy5- and Cy3labeled probes and a mix of COT-1 and Herring sperm DNA were ethanol-precipitated at $-80^{\circ} \mathrm{C}$ for at least $30 \mathrm{~min}$. Hybridization was done using dissolved precipitated probes in a hybridization buffer. 
Arrays were washed in sodium chloride-sodium citrate (SSC) buffers with decreasing concentrations and scanned by a GenPix 4100A (Axon Instruments, Union City, CA, USA). The images were analyzed by BlueFuse for Microarrays 3.5 software (BlueGnome). In data processing $\log _{2}$ ratios of $\mathrm{Cy} 3$ and $\mathrm{Cy} 5$, intensities were generated for all hybridized clones. Normal copy numbers were considered to be present if the $\log _{2}$ ratio was between -0.3 and +0.3 , values above +0.3 were interpreted as gain/ amplification and those under -0.3 as losses (deletions). Genomic profiles were represented plotting $\log _{2}$ ratios in Y-axis and the 23 chromosomes in Xaxis. Individual chromosomal profiles were represented with clone positions in Y-axis and $\log _{2}$ ratios in $\mathrm{X}$-axis.

\section{RESULTS}

We studied blood from our four patients by the method of array-CGH with CytoChip (BlueGnome), covering all autosomes and sex chromosomes at a mean density of $1 \mathrm{BAC}$ clone $/ 0.5 \mathrm{Mb}$. More than $95 \%$ of genomic clones were successfully hybrid- ized in each case. Standard deviation in $\log _{2}$ ratios of Cy3 and Cy5 intensities [test $(\mathrm{T})$ and normal $(\mathrm{N})$ DNA, respectively] ranged between 0.03 and 0,07 , depending on the quality of DNA.

We used two approaches to identify BACs that showed significant loss or gain in the analyzed samples: a) observation of high-level $\operatorname{losss}_{(} \log _{2} \mathrm{~T}: \mathrm{N}$ ratio $<-0.5)$ and of high-level gain $\left(\log _{2} \mathrm{~T}: \mathrm{N}\right.$ ratio $\left.>+0.5\right)$ and $b$ ) detection of at least one additional adjacent clone with the same aberration in the same probe. The single aberrant clones were excluded from analysis. Using the data base of BlueFuse (BlueGnome) we determined the copy number polymorphisms in the patients.

In patient 1, we found no specific micro-abnormality, but discovered a polymorphism in the following loci: 2p25.3, 4p15.1, 10q11.21, 10q26.3, 11q13.4, 16p12.1, 17q21.31, 19p13.2, 19q13.33 and Yp11.2 (Figure 1). Their population frequency varied between $1-60 \%$ according to the BlueGnome data base.

In patient 2, no specific abnormalities were found but seven loci showed a variable number of gene copies (polymorphic regions) in the follow-

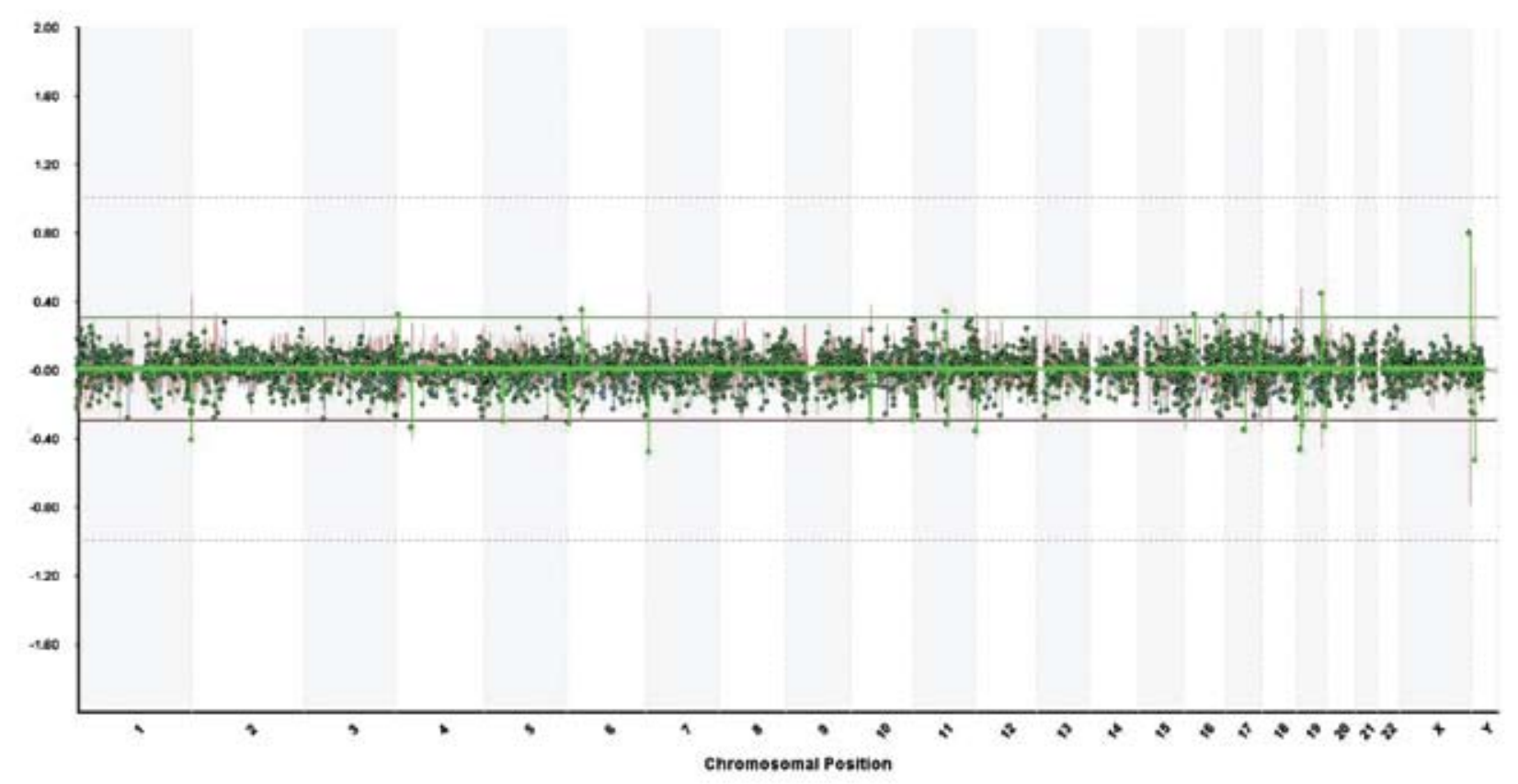

Figure 1. Genomic profile across all chromosomes in patient 1. X-axis: genomic clones on chromosomes $1-22, \mathrm{X}$ and Y; Y-axis: $\log _{2}$ ratio of test to normal DNA for each clone. 


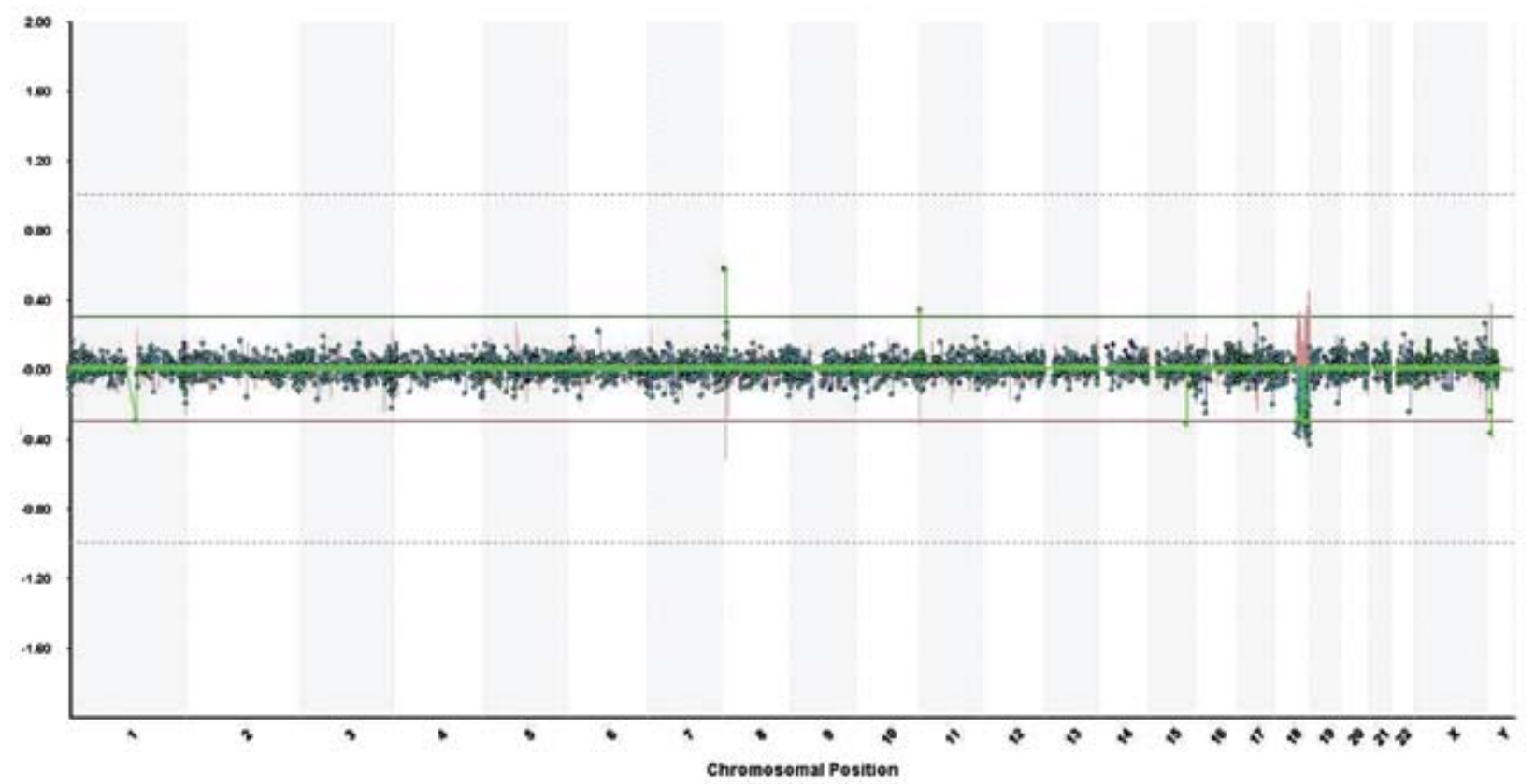

Figure 2. Genomic profile across all chromosomes in patient 3, showing deletion 18q. X-axis: genomic clones on chromosomes 1-22, $\mathrm{X}$ and $\mathrm{Y}$; Y-axis: $\log _{2}$ ratio of test to normal DNA for each clone.

ing positions: $1 \mathrm{p} 13.2,1 \mathrm{q} 21.3,1 \mathrm{q} 44,2 \mathrm{p} 16.1,3 \mathrm{q} 29$, $4 \mathrm{p} 16.1$ and $5 \mathrm{q} 13.2$.

In patient 3 , array-CGH analysis revealed genomic loss in the long arm of chromosome 18, which, on the basis of the value of $\log _{2}$ ratio, was interpreted as a mosaic form (Figure 2). The deletion comprised a DNA sequence from $18 \mathrm{q} 21.1$ to $18 \mathrm{q} 23$ (about $28 \mathrm{Mb}$ ) (Figure 3). Beside the deletion, we identified gene copy polymorphisms in positions 8p23.1, 10q26.3 and Yp11.2 On the basis of the presence of the large deletion 18q21-23, we repeated cytogenetic analyses in 100 cells and found a low frequency of mosaicism: 46, XY (83\%)/46,XY,del (18)(q21-qter) (Figure 4).

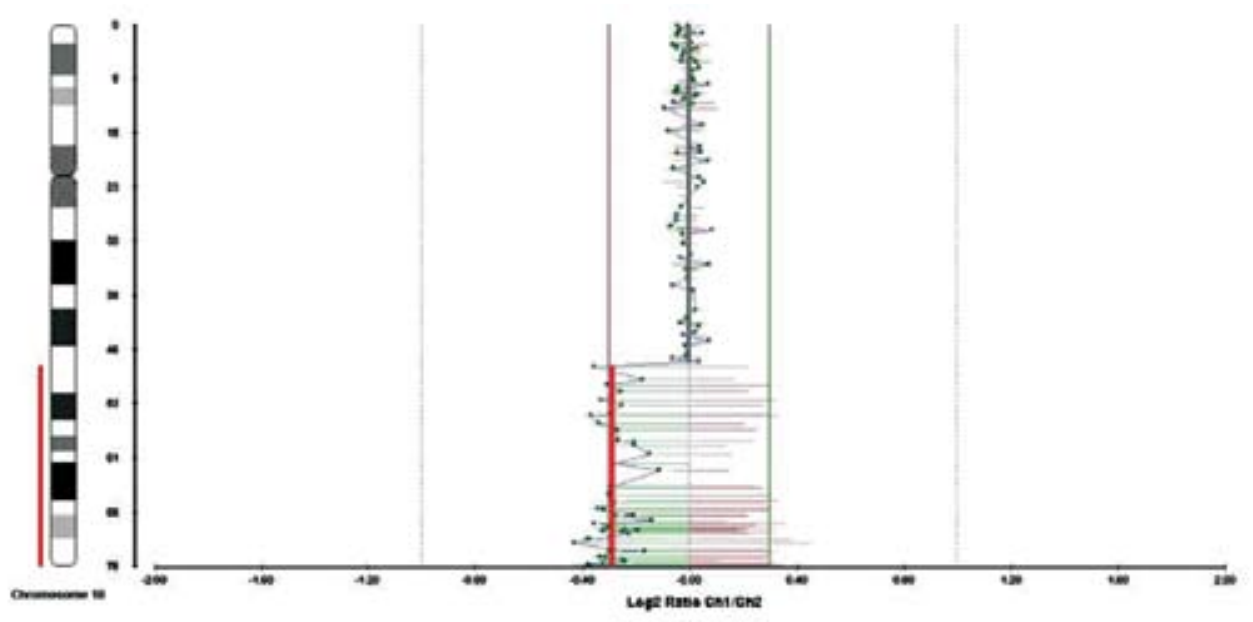

Figure 3. Genomic profile of patient 3 for chromosome 18, revealing deletion of 18q21-23. X- $\log _{2}$ ratio of test to normal DNA for clones on chromosome 18; Y-axis: genomic clones of chromosome 18 . 
A
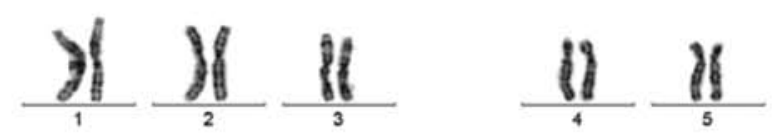

If $\frac{26}{2} \frac{\| 1}{6} \frac{\| 1}{10} \frac{\| 11}{11}$
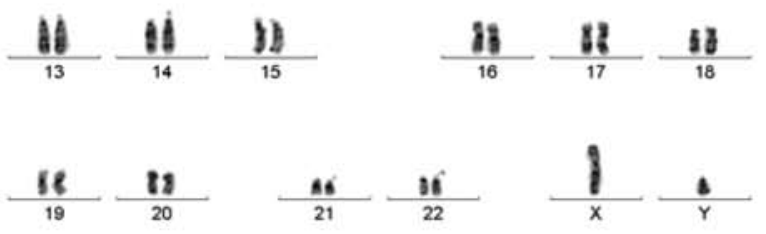

B

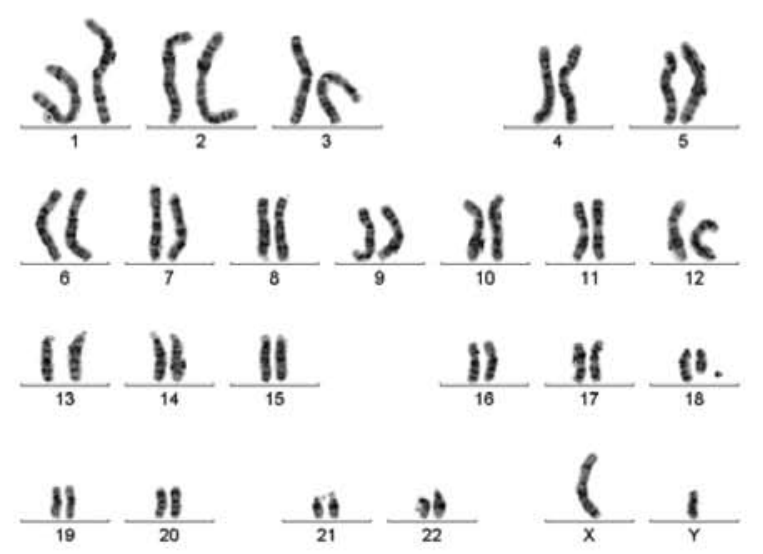

Figure 4. Karyotype of patient 3. A: normal cell line 46,XY; B: abnormal karyotype with the 18q deletion.

In patient 4, we established copy number polymorphisms in the loci $1 \mathrm{p} 31.1,4 \mathrm{p} 16.1$ and 8p23.2.

\section{DISCUSSION}

We used the technique of array-CGH to screen micro-structural whole genome copy number changes in four patients with congenital malformations. We detected a specific genomic abnormalities in one of the four patients and different copy number polymorphisms in all four patients.

This technique permits identification at a high resolution of micro-structural abnormalities in human chromosomes [7]. In comparison to fluorescent in situ hybridization (FISH) and classical cytogenetic diagnostics, the method has a much higher sensitivity and permits screening of the whole genome simultaneously for unbalanced micro-structural rearrangements [8]. It is also applicable for effective screening of new dose-dependent genes, which are important for the emergence of many human diseases [9]. The use of the BAC clones ensures strong and large signals that can be measured over a broad range - from deletions to amplifications $[9,10]$.

We established copy number polymorphisms in all of our patients. According to recent publications [11-13], more than half of the variability between human genomes is due to copy number variations (CNVs) of small regions of DNA. It is hypothesized that these CNVs are responsible for some complex diseases and are more frequent than single nucle- otide polymorphisms [11-13]. There are more than 6,000 known regions of $\mathrm{CNV}$, and there may be many more $[13,14]$. The impact of CNVs in the development of congenital malformations remains to be seen.

Our most interesting finding was the loss of genetic material from chromosome 18 in patient 3 . This deletion comprises the region 18q21.1-q23 and encompassed 48 deleted BAC clones. The $18 \mathrm{q}$ syndrome (OMIM \#601808) is characterized by mental retardation, facial dysmorphism, congenital malformations and deformities of the feet, depending on the size of the deleted region [15]. The critical region in the 'typical' $18 \mathrm{q}$ phenotype is one of $4.3 \mathrm{Mb}$ located within 18q22.3-q23. A recent study investigated partial deletions of the long arm of chromosome 18 and identified critical regions for microcephaly (18q21.33), short stature (18q21.-q21.33, and 18q22.3-q23), white matter disorders (18q22.3-q23) and CAA (congenital aural atresia) (18q22.3) [15]. These observations are consistent with the clinical symptoms in our patient. During investigation of the deleted region in the long arm of chromosome 18 by genome browser, we delineated 149 genes, of which some have no known function. Other genes in this region may also be related to the abnormal phenotype. Among these we found genes with regulatory function (ZNF236, zinc finger protein 236), transcription coactivators and regulators [DCC, deleted in colorectal carcinoma; RAX, retina and anterior neural fold homeobox; TSHZ1, teashirt family zinc finger 1), translation regulators 
(NARS, asparaginyl-tRNA synthetase), transporters (CCBE1, collagen and calciumbinding EGF domains 1), inducte of apoptosis (PMAIP1, phorbol12-myristate-13-acetate-induced protein 1), regulators of cell growth (SOCS6, suppressor of cytokine signaling 6; PARD6G, par-6 partitioning defective 6 homolog gamma (C. elegans)] and others. These genes may be good candidates for further functional studies, as candidate genes for the human development and congenital anomalies. Our concurrent array $\mathrm{CGH}$ analysis and karyotyping in the patient with $18 \mathrm{q}$ deletion, showed that microarray technology is useful for the detection of low level mosaicism.

\section{CONCLUSIONS}

We have used the method of array-CGH on DNA from four patients with "mental retardation of unclear etiology" and have analyzed whole genome copy number at a high resolution. We detected different copy number polymorphisms in all patients. We have identified a mosaic form of deletion $18 \mathrm{q} 21.1-\mathrm{q} 23$ in one of the patients.

The use of array-CGH technology increases our understanding of the normal quantitative variants of the human genome and makes possible determination of the exact size and the boundaries of the deletion in patients. We could thus obtain the precise information necessary for adequate genetic counseling of the families affected by various aneuploidies.

\section{ACKNOWLEDGMENTS}

This study was supported by Contract No. 5/02.05. 2007 of the Committee of Medical Sciences, Medical University Sofia, Sofia, Bulgaria and the Infrastructural Project of the Ministry of Education and Science, Sofia, Bulgaria (contract No. 05/01.08.2005).

\section{REFERENCES}

1. Nikkila A, Kallen B, Marsal K. Fetal growth and congenital malformations. Ultrasound Obstet Gynecol 2007; 29(3): 289-295.

2. Verrotti C, Caforio E, Gramellini D, Nardelli GB. Ultrasound screening in second and third tri- mester of pregnancy: an update. Acta Biomed 2007; 78(3): 229-232.

3. Brent RL. Environmental causes of human congenital malformations: the pediatrician's role in dealing with these complex clinical problems caused by a multiplicity of environmental and genetic factors. Pediatrics 2004; 113(Suppl 4): 957-968.

4. Oberlander TF, Warburton W, Misri S, Riggs W, Aghajanian J, Hertzman C. Major congenital malformations following prenatal exposure to serotonin reuptake inhibitors and benzodiazepines using population-based health data. Birth Defects Res B Dev Reprod Toxicol 2008; 83(1): 68-76.

5. Botto LD, Mastroiacovo P. Exploring genegene interactions in the etiology of neural tube defects. Clin Genet 1998; 53(6): 456-459.

6. Smeets DF. Historical prospective of human cytogenetics: from microscope to microarray. Clin Biochem 2004; 37(6): 439-446.

7. Pollack JR, Perou CM, Alizadeh AA, Eisen MB, Pergamenschikov A, Williams CF, Jeffrey SS, Botstein D, Brown PO. Genome-wide analysis of DNA copy-number changes using cDNA microarrays. Nat Genet 1999; 23(1): 41-46.

8. Bejjani BA, Shaffer LG. Application of arraybased comparative genomic hybridization to clinical diagnostics. J Mol Diagn 2006; 8(5): 528-533.

9. Albertson DG, Pinkel D. Genomic microarrays in human genetic disease and cancer. Hum Mol Genet 2003; 12(Spec No 2): 145-152.

10. Pinkel D, Segraves R, Sudar D, Clark S, Poole I, Kowbel D, Collins C, Kuo WL, Chen C, Zhai Y, Dairkee SH, Ljung BM, Gray JW, Albertson DG. High resolution analysis of DNA copy number variation using comparative genomic hybridization to microarrays. Nat Genet 1998; 20(2): 207-211.

11. Freeman JL, Perry GH, Feuk L, Redon R, McCarroll SA, Altshuler DM, Aburatani H, Jones KW, Tyler-Smith C, Hurles ME, Carter NP, Scherer SW, Lee C. Copy number variation: new insights in genome diversity. Genome Res 2006; 16(8): 949-961.

12. McCarroll SA, Altshuler DM. Copy-number variation and association studies of human disease. Nat Genet 2007; 39(Suppl 7): S37-42.

13. Redon R, Ishikawa S, Fitch KR, Feuk L, Perry GH, Andrews TD, Fiegler H, Shapero MH, Carson AR, Chen W, Cho EK, Dallaire S, Freeman JL, González JR, Gratacòs M, Huang J, Kalaitzo- 
poulos D, Komura D, MacDonald JR, Marshall CR, Mei R, Montgomery L, Nishimura K, Okamura K, Shen F, Somerville MJ, Tchinda J, Valsesia A, Woodwark C, Yang F, Zhang J, Zerjal T, Zhang J, Armengol L, Conrad DF, Estivill X, Tyler-Smith C, Carter NP, Aburatani H, Lee C, Jones KW, Scherer SW, Hurles ME. Global variation in copy number in the human genome. Nature 2006; 444(7118): 444-454.
14. Carter NP. Methods and strategies for analyzing copy number variation using DNA microarrrays. Nat Genet 2007; 39(Suppl 7): S16-21.

15. Feenstra I, Vissers LE, Orsel M, van Kessel AG, Brunner HG, Veltman JA, van RavenswaaijArts CM. Genotype-phenotype mapping of chromosome $18 \mathrm{q}$ deletions by high-resolution array CGH: an update of the phenotypic map. Am J Med Genet 2007; 143(16): 1858-1867. 
